\title{
AVALIAÇÃO DO CURSO DE PANIFICAÇÃO OFERTADO PELO PROGRAMA DE EXTENSÃO GASTRONOMIA SOCIAL
}

FERREIRA. E.T. ${ }^{1}$, BASTOS, M.S. ${ }^{2}$, T.GARCEZ, C.N.A. ${ }^{3}$, MOTA, R.N. ${ }^{4}$, AMARAL, Q.G. ${ }^{5}$ \& COSTA, E.A. ${ }^{6}$

${ }^{1}$ Bolsista e graduando pela Universidade Federal do Ceará (UFC). E-mail: etf1974@ hotmail.com, ${ }^{2}$ Servidora técnico-administrativa da Universidade Federal do Ceará (UFC). E-mail: reginabastos_ufc@yahoo.com.br; ${ }^{3}$ E-mail: terezacarla@ hotmail.com; ${ }^{4}$ Professor da

Universidade Federal do Ceará. E-mail: motarobson@gmail.com, ${ }^{5}$ E-mail: rafaelgurgel.amaral@ gamil.com; ${ }^{6}$ Professor da Universidade Federal do Ceará e coordenador da ação extensionista de mesmo nome do artigo. E-mail: evelinedealencar@ufc.br

DOI: https://doi.org/10.32356/exta.v2.n18.31247 - Artigo submetido em 12/02/2018

\section{RESUMO}

O presente artigo visa apresentar o Curso de Panificação básica e a avaliação realizada pelos participantes. $\mathrm{O}$ mesmo ocorreu durante o mês de julho de 2017 na Escola de Gastronomia Autossustentável, localizada no bairro do Bom Jardim, por meio do programa de extensão Gastronomia Social em parceria com o Movimento de Saúde Mental Comunitária e a Escola de Gastronomia Autossustentável. O programa de extensão trabalha com a qualificação de jovens e adultos, de preferência da comunidade do Bom Jardim, a fim de incentivar a inserção no mercado de trabalho através da qualificação, além de despertar a discussão que envolve a gastronomia como arte, cultura e ciência. Dentro desse programa, o Curso de Panificação foi pensado para ensinar os conceitos básicos da teoria da panificação e a aplicação na cozinha, visando apresentar os ingredientes utilizados na panificação e suas funções e as diferentes técnicas na produção de pães. Portanto, o feedback recebido pelos alunos é de grande relevância para a melhoria da didática e até mesmo do planejamento dos cursos oferecidos, a fim de atender a demanda da comunidade.

PALAVRAS-CHAVE: Gastronomia Social. Panificação. Qualificação.

\section{EVALUATION OF THE BAKERY COURSE OFFERED BY THE SOCIAL GASTRONOMY EXTENSION PROGRAM}

\begin{abstract}
This article aims to present the bakery Course basic and evaluation by participants. The same occurred during the month of July 2017 at school of Sustainable Cuisine, located in the neighborhood of Bom Jardim, through the Social Food outreach program in partnership with the Community Mental Health movement and school of Sustainable Cuisine. The outreach program works with the qualification of young people and adults, preferably Good community garden, in order to encourage the integration into the labour market through training, in addition to arouse discussion involving the food as art, culture and
\end{abstract}

science. Within this program, the bakery Course was designed to teach the basic concepts of the theory of the bakery and the application in the kitchen, in order to present the ingredients used in baking and its functions and the different techniques in the production of bread. Therefore, the feedback received by the students is of great importance for the improvement of teaching and even the planning of the courses offered in order to meet the demand of the community.

KEYWORDS: Social Gastronomy. Bakery. Qualification.

\section{INTRODUÇÃO}

O Programa de Extensão Gastronomia Social criado em 2010 surgiu para a atender a necessidade da comunidade como um agente de transformação social. Tal ação busca por meio da realização de cursos e oficinas gratuitos unir outros fatores também importantes para a capacitação como a ciência, arte e cultura sobre aquilo que está sendo produzido pelos alunos, 
o resgate da memória gastronômica, seus hábitos e tradições. Além disso, visa trabalhar a inserção de jovens e adultos no mercado de trabalho e ou incentivá-los a terem seu próprio empreendimento. (COSTA, 2012; COSTA, 2013; CUNHA et al., 2015; FROTA et al. 2015; LEVIEN et al. 2015)

Outra prerrogativa importante que fundamentou o surgimento desta ação extensionista foi o fato de proporcionar aos graduandos em Gastronomia da UFC a experiência com a docência, por meio da condução de aulas teóricas e práticas. Portanto, os personagens que conduzem o mesmo são: os professores (graduandos do Bacharelado em Gastronomia da UFC, ou seja, bolsistas remunerados, extensionistas voluntários e estagiários), os monitores (também graduandos em Gastronomia da UFC, porém recém ingressos na ação, os quais auxiliaram na condução das aulas teóricas e práticas) e os coordenadores da ação (Professores do Curso de Gastronomia da UFC, que planejam os cursos e supervisionam as atividades dos "alunos/professores").

A Escola de Gastronomia Autossustentável (EGA) inaugurada em 2016 faz parte do Programa de Extensão da UFC e trata-se de um espaço de formação construído e implementado pelo Movimento de Saúde Mental Comunitária do Bom Jardim (MSMC). A EGA foi também pensada em ser um espaço para a sustentabilidade das ações psicoterapeutas do MSMC como uma forma de facilitar o equilíbrio e a saúde mental, a evolução social e comunitária das pessoas, bem como a profissionalização das pessoas que passam pelo Programa. (COSTA et al., 2016).

O Gastronomia Social também possui outros parceiros, que geram demandas de atividades para a realização e cursos de gastronomia, tais como o Centro Cultural do Bom Jardim (CCBJ), a Secretaria de Educação - Coordenadoria do EJA com qualificação e a Escola de Vida Arte e Sabor - EVISA ambos do Governo do Estado do Ceará, a Unidade Universitária Núcleo de Desenvolvimentos da Criança (UUNDC) da UFC, o IFCE Campus avançado de Camocim, o Projeto Realimenta e o Instituto da Primeira Infância - IPREDE; a Biblioteca Herbênia Gurgel da Prefeitura de Fortaleza e o projeto Colcha de Retalhos ligada à Rede Solidárias no Grande Bom Jardim (COSTA, 2019).

O referido Programa já ofertou mais de 35 cursos (com diferentes temáticas e carga horária) à população do Grande Bom Jardim e comunidade em geral, atingindo de forma indireta mais de duas mil pessoas (COSTA, 2019). A preferência de atendimento aos bairros 
que compõe a área do grande Bom Jardim (Canidezinho, Granja Lisboa, Granja Portugal, Siqueira e Bom Jardim) deve-se a situação de vulnerabilidade social que os mesmos enfrentam, com baixo IDH e altos índices de violência (HOLANDA, 2013). Portanto, esse atendimento visa estimular e incentivar a entrada de jovens e adultos no mercado de trabalho, ao universo empreendedor e também despertar a discussão que envolve a gastronomia como arte, cultura e ciência.

A panificação é uma ciência que visa desenvolver a arte e as técnicas aplicadas no desenvolvimento de pães e de outras massas alimentícias e outras preparações ligadas a panificação. O pão é um alimento simples produzido a base de farinha, água, sal e fermento. Porém para obter resultados satisfatórios é necessário conhecer cada ingrediente, sua função na produção e seu comportamento diante das técnicas aplicadas (SEBESS, 2010).

Relata-se que o pão é um alimento histórico com produção e consumo (sem fermentação) relatados por volta do século 11 a.C. na Europa e no Egito antigo, há relatos do uso da massa fermentada. É um alimento bastante consumido e com grande simbolismo cultural por estar presente em várias religiões e na base alimentar de muitos povos (KÖVESI, 2011).

O Curso de Panificação é ofertada pelo Programa Gastronomia Social devido ao interesse da comunidade pelo conteúdo do mesmo, tendo sido ofertado em outras edições e com outros parceiros, porém com cargas horárias diferentes (FERREIRA et al., 2017).

O presente artigo visa apresentar o Curso de Panificação Básica ofertado pelo Programa de Extensão Gastronomia Social no Jardim da Gente, da Universidade Federal do Ceará e a avaliação realizada pelos participantes. O mesmo ocorreu na Escola de Gastronomia Autossustentável, localizada no bairro do Bom Jardim, Fortaleza-CE, e pertence ao Movimento de Saúde Mental Comunitária.

\section{MATERIAIS E MÉTODOS}

O Curso de Panificação Básica avaliado neste estudo compreende a primeira turma dessa temática formada na Escola de Gastronomia Autossustentável (EGA), em julho de 2017, com carga horária de 40h/a e foi totalmente gratuito. O objetivo geral do curso foi apresentar e ensinar a panificação e suas diversas técnicas, ingredientes e funções em um viés básico a fim de cumprir com a função de capacitação pelo Gastronomia Social na comunidade.

Dentro desse contexto que envolve o Gastronomia Social o Curso de Panificação básica 
foi planejado em dois módulos:

I - Higiene e manipulação dos alimentos, ergonomia e racionalização do trabalho; elaboração de ficha técnica, controle de custos e economia criativa; história da panificação, função dos ingredientes na composição de um produto, classificação e seleção dos ingredientes da panificação, técnicas de batimento e fermentação.

II - Panificação básica: aplicação prática dos assuntos abordados nas aulas anteriores como: pesagem, sovagem, boleamento, modelagem, corte e assamento; execução das fichas técnicas e compreensão sobre os diferentes tipos de pães: massas magras, macias, ricas, doces e úmidas; discussão sobre a cultura e consumo do pão no Ceará e a influência desse alimento.

A presente pesquisa de campo, de abordagem dedutiva, descritiva e classificada quanto à natureza como um trabalho científico original conforme Andrade (2010).

A pesquisa foi realizada no período de julho de 2017 com os 23 alunos participantes do Curso de Panificação ofertado pelo Programa de Extensão Gastronomia Social no Jardim da Gente da UFC. A hipótese motivadora do estudo foi baseada em responder a pergunta de investigação: o Curso de Panificação atende a demanda dos participantes? Ou seja, acreditando ser uma ferramenta de mudança social e inserção no mercado de trabalho?

A ferramenta utilizada para a avaliação foi o questionário estruturado em oito perguntas aplicado junto aos participantes ao final do Curso (precisamente no último dia de atividade). As perguntas foram objetivas e tinham opções de respostas para múltiplas escolhas, porém apenas uma teria que ser marcada.

A avaliação foi centrada nas seguintes interrogações acerca dos aspectos didáticos, feedback sobre a expectativa do curso e divisão das atividades teóricas e práticas, avaliação da estrutura do ambiente de realização e outros aspectos:

- O professor demonstrou domínio suficiente dos conteúdos abordados?

- Houve uma sequência de assunto para facilitar o aprendizado?

- As técnicas de ensino foram adequadas?

- O curso corresponde as suas expectativas?

- A carga horária foi adequada?

- A divisão de aulas teóricas e práticas foi adequada?

- A quantidade de participantes foi adequada? 
- A infraestrutura foi adequada?

Os dados foram analisados utilizando Programa Excel $2013^{\circledR}$ e expressos em percentual a partir da frequência obtida.

\section{RESULTADOS E DISCUSSÃO}

A seguir serão apresentados os resultados da avaliação do Curso de Panificação do Programa Gastronomia Social da UFC. É possível verificar que todas as perguntas obtiveram respostas positivas, porém tornou-se necessário avaliar individualmente cada uma.

Quanto aos aspectos didáticos observa-se que os professores demonstraram domínio dos conteúdos ministrados, que as técnicas de ensino foram adequadas e as sequências de assuntos facilitaram o aprendizado (Figura 1).

FIGURA 1 - Avaliação dos aspectos didáticos do Curso de Panificação

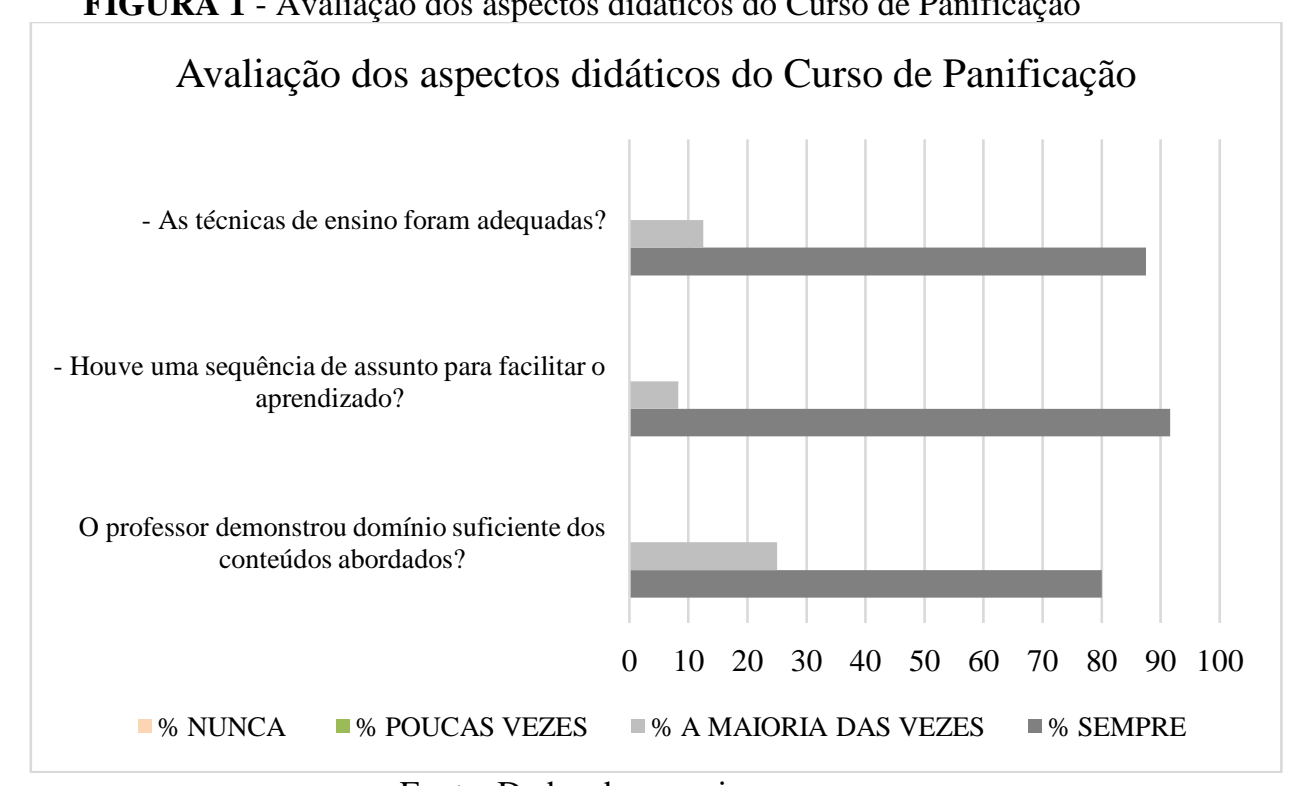

Fonte: Dados da pesquisa.

Os resultados obtidos foram reflexo do planejamento das aulas e das reuniões com a coordenação do Programa de Extensão Gastronomia Social, pois nestas eram repassadas orientações para o melhor desenvolvimento didático das aulas teóricas e práticas. Os professores seguiam o plano de curso e as instruções didáticas apresentadas aos respectivos conteúdos programáticos, porém estavam livres para sugerir atividades, dinâmicas de grupo ou até mesmo a condução ou não do trabalho entre equipes.

A direção de uma sala de aula reflete muito no processo de aprendizagem dos indivíduos 
e a atividade do professor é uma das modalidades específicas que interfere na sociedade (LIBÂNEO, 2017). Segundo Gomes, Moreira e Pinheiro (2018) a metodologia comumente utilizada na sala de aula ainda é baseada em tradicionalismo, onde o professor é um mero transmissor de conhecimentos, entretanto, novos métodos, os quais inovadores (metodologias ativas, ou uso de recursos eletrônicos, informáticos e outros), estão sendo utilizados a fim de obter um ensino de qualidade. Lorenzato (2008) alega que ministrar aulas é diferente de ensinar, pois este proporciona condições para que o aluno construa seu próprio conhecimento; e ainda ressalta sobre a compreensão se houve de fato um ensino quando ocorre aprendizagem. O autor alega que existe a possibilidade de se dar aula sem conhecer, mas não há possibilidade de se ensinar sem conhecer, sem desenvolver uma didática para um determinado conteúdo a fim de conferir aprendizagem significativa.

Quanto ao feedback recebido sobre a expectativa do Curso de Panificação (Figura 2) 80\% responderem que sim e $88 \%$ afirmaram que as aulas teóricas e práticas tiveram divisões adequadas. Este resultado é relevante para os organizadores, pois relevam se o objetivo proposto pelo Curso oferecido foi atingido. No caso do conteúdo de panificação o plano de curso objetivava capacitar os participantes para desenvolverem técnicas básicas na elaboração de massas simples e para a inserção no mercado de trabalho, por meio de aulas teóricas e práticas.

FIGURA 2 - Expectativa do curso e a divisão das atividades teóricas e práticas do Curso de Panificação.

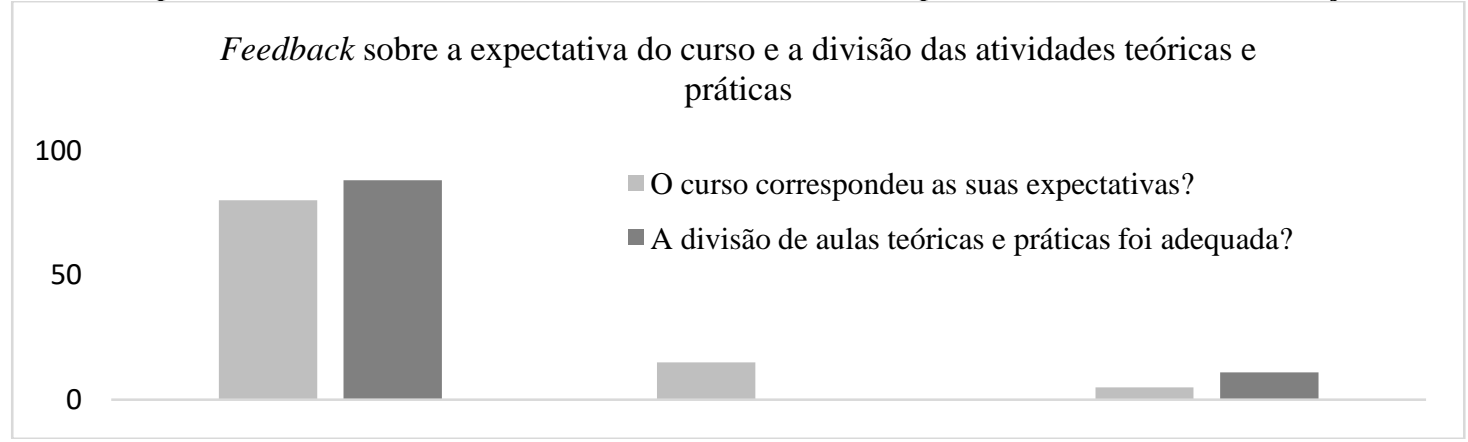

Fonte: Dados da pesquisa.

O resultado obtido revelou que as aulas teóricas e práticas atenderam as expectativa. É possível observar que a divisão da carga horária entre os dois tipos de aulas permitiram aos alunos se desenvolverem com os demais e com o conteúdo apreendido, pois se apropriaram do conhecimento que receberam. De acordo com Le Boterf (2003) a aprendizagem coletiva 
promove um intercâmbio de conhecimentos e experiências individuais voltado à aprendizagem coletiva e promove ainda interações afetivas que permitem aos membros se sentirem à vontade e terem prazer no trabalho em conjunto.

Para Vieira et al.(2013) as atividades práticas são eficientes, facilitadoras do processo ensino aprendizagem, porém devem ser ter seu planejamento antecipado serem relacionadas com os conteúdos teóricos ministrados. Os alunos demonstraram grande interesse pelas aulas práticas, pois promovem maior participação, integração e rendimento da aprendizagem.

No que se refere a carga horária do Curso avaliado (Figura 3) observa-se que $93 \%$ dos participantes classificou como adequada, ou seja, o planejamento do número de dias a fim de atingir o total de 40h/a foi plenamente alcançado. Portanto, sugere-se a manutenção da mesma para futuras turmas.

FIGURA 3 - Adequação da carga horária do Curso de Panificação

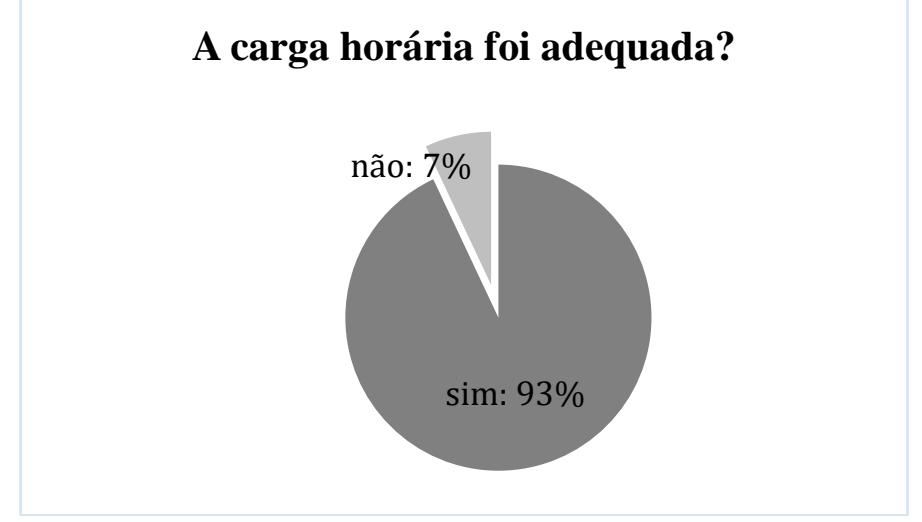

Fonte: Dados da pesquisa.

De acordo com o Ministério da Educação (MEC) podemos classificar o referido Curso de Panificação, objeto desse estudo, como "curso de livre oferta", pois não há carga horária préestabelecida, além disso enquadra-se na seguinte caracterização: modalidade aberta à comunidade, com suas matrículas condicionadas à capacidade de aproveitamento da formação, e não necessariamente ao nível de escolaridade; podem apresentar características diversificadas em termos de preparação para o exercício profissional, por exemplo para algumas ocupações básicas ou relacionadas ao exercício pessoal de atividades geradoras de trabalho e renda (BRASIL, 2018).

Ao interrogar se o número de participantes (Figura 4), ou seja, se o tamanho da turma foi adequado, verificou-se que a maioria (54\%) declararam como boa. Entende-se que o número 
de alunos influencia no processo de didática e da aprendizagem, pois a atenção junto aos mesmos, principalmente durante as tarefas práticas pode ser comprometidas. Portanto, turmas pequenas facilitam o trabalho prático, a comunicação, o acompanhamento dos alunos e a supervisão das tarefas. Além disso, promovem o desenvolvimento de competências coletivas, as quais procedem das relações de cooperação e sinergia entre os integrantes das equipes. As competências coletivas abrangem: a consciência que os membros têm sobre as atividades a serem desenvolvidas na direção de um objetivo comum, ocorrendo ainda interações afetivas, aprendizagem coletiva, sentido coletivo, base de conhecimento comum, senso de interdependência de tarefas e referencial comum, linguagem compartilhada e engajamento.

FIGURA 4 - Avaliação do tamanho da turma e ou número de alunos do Curso de Panificação estudado A quantidade de participantes foi adequada?

- ruim: $0 \%$

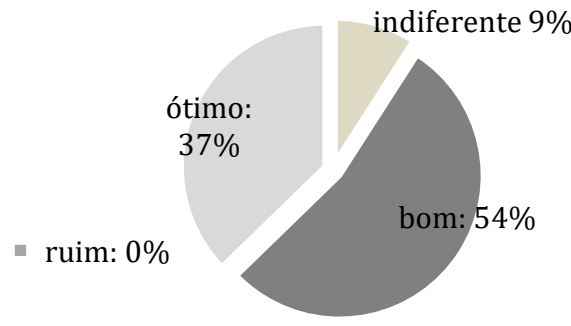

Fonte: Dados da pesquisa.

Giansante e colaboradores (2015) avaliaram a influência das Competências Coletivas (CCs) sobre o desempenho coletivo em equipes de gastronomia (compostas por um total de 131 estudantes de um curso superior de Tecnologia em Gastronomia, de uma Universidade particular do Estado de São Paulo) e verificaram que todas as equipes estudadas desenvolveram tais competências durante a execução das tarefas. Os aurores observaram que os integrantes eram contribuintes na execução da tarefa para atingirem a meta dada durante as aulas práticas.

Quanto a avaliação da estrutura do espaço onde as aulas foram realizadas (EGA), 90\% alegaram ser adequada. A EGA foi planejada para atender atividades práticas do Programa Gastronomia Social. A mesma é composta por quatro praças de trabalho, dotadas com fogões, pias e bancadas. $\mathrm{O}$ espaço conta com utensílios e equipamentos como refrigeradores, freezer, forno elétrico industrial, batedeiras, liquidificadores e outros. A mesma possui sistema de exaustão a fim de proporcionar conforto térmico durante as atividades práticas. (COSTA et al., 
2016). As aulas teóricas são ministradas em sala de aula no andar superior da EGA, dotadas com recursos audiovisuais.

Diante do exposto verifica-se que as instalações da EGA proporcionam a execução do Curso de Panificação planejado e para o número de alunos participantes, sem comprometer o processo ensino-apredizagem. A estrutura encontra-se dentro dos padrões da RDC nº 216/2004 (BRASIL, 2004) que regulamenta as boas práticas para unidades produtoras de alimentos. A EGA foi planejada fisicamente para atender cursos do Programa Gastronomia Social no Jardim da Gente e os equipamentos e utensílios também foram adquiridos para esta finalidade, conforme Costa (2019).

\section{CONCLUSÃO}

Conclui-se que o Curso de Panificação ofertado pelo Programa Gastronomia Social no Jardim da Gente da UFC é de grande relevância para a comunidade, tendo professores com domínio do conteúdo e espaço adequado para a realização das aulas práticas. O curso também atende as expectativas dos alunos e, portanto, sugere-se a oferta de outras turmas à comunidade. Além disso, a presente ação extensionista foi relevante para aproximar da docência os graduandos em Gastronomia e permitir a troca de saberes com os participantes, proporcionando assim a inclusão e a profissionalização dentro da comunidade.

Portanto, o número de participantes, a carga horária desenvolvida, a divisão didática do conteúdo entre aulas teóricas e práticas do Curso de Panificação foram bem avaliados pelos alunos, bem como a metodologia didática desenvolvida pelo professor e a infraestrutura, pois atenderam às expectativas. Pode-se concluir, ainda, que o referido Curso promoveu o aprendizado e o desenvolvimento de competências coletivas.

O feedback recebido pelos alunos é de grande relevância para a melhoria da didática e até mesmo para o planejamento dos demais cursos oferecidos pelo Programa de Extensão Gastronomia Social no Jardim da Gente.

\section{REFERÊNCIAS}

ANDRADE, M. M. de. Introdução a metodologia do trabalho científico: elaboração de trabalhos na graduação. São Paulo: Editora Atlas S. A., 2010. 
BRASIL. Ministério da Educação. Cursos de Formação Inicial e Continuada (FIC) ou Qualificação Profissional. Brasília, 2018. Disponível em: <http://portal.mec.gov.br/cursos-daept/formacao-inicial-e-continuada-ou-qualificacao-profissional>. Acesso em: 15 fev. 2020.

. Ministério da Saúde. Agência Nacional de Vigilância Sanitária. Resolução RDC no 216 de 15 de setembro de 2004. Dispõe sobre as boas práticas para serviço de alimentação. Brasília, DF, 162 set. 2004.20 Disponível <http://elegis.anvisa.gov.br/leisref/public/showAct.php?id=12546>. Acesso em: 15 fev. 2020.

COSTA, E. A. de. Escola de Gastronomia AME. Formulário de Cadastramento de Programa de Extensão. Fortaleza: Universidade Federal do Ceará, 2019.

Programa gastronomia social no jardim da gente. Formulário de Cadastramento de Programa de Extensão. Fortaleza: Universidade Federal do Ceará, 2019.

COSTA, E. A. Programa de Gastronomia oferece cursos gratuitos de culinária cearense e doces. Fortaleza, 2013. Disponível em: $<$ http://www.ufc.br/noticias/noticias-de-2013/4139Programa-de-extensao-de-gastronomia-oferece-cursos-gratuitos-de-culinaria-cearense-edoces>. Acesso em: 06 jan. 2014.

COSTA, E. A.; FREIRE, V. N.; BASTOS, R. M. S. ; MARTINS, N. ; BONVINI, O. ; CAETANO, D. R. A. ; AMARAL, R. G. ; DUMMAR, R. . Escola de Gastronomia da Comunidade do Grande Bom jardim como ação do Programa Gastronomia Social. Rev. Enc. Univ. da UFC, v. 01, p. 3792-3792, 2016.

CUNHA, Elba Cristina Barbosa da; COSTA, Eveline de Alencar; COSTA, Daniel; MOTA, Robson Nascimento; OLIVEIRA, Patrícia de Freitas; BASTOS, Regina Maria Silva. Gastronomia Saudável em Camocim - CE como Ação da Semana Mundial da Alimentação. In: XXIV Encontro de Extensão. Encontros Universitários 2015. Fortaleza, Anais... Fortaleza: UFC, 2015. Gastronomia social no jardim da Gente. Disponível em: <https://www.facebook.com/GastronomiaSocialNoJardimDaGente?fref=ts>. Acesso em: 06 jan. 2014.

CUNHA, Elba Cristina Barbosa da; COSTA, Eveline De Alencar; COSTA, Daniel; MOTA, Robson Nascimento; OLIVEIRA, Patrícia de Freitas; BASTOS, Regina Maria Silva. Gastronomia Saudável em Camocim - CE como Ação da Semana Mundial da Alimentação. In: XXIV Encontro de Extensão. Encontros Universitários 2015. Fortaleza, Anais... Fortaleza: UFC, 2015. Gastronomia social no jardim da Gente. Disponível em: <https://www.facebook.com/GastronomiaSocialNoJardimDaGente?fref=ts>. Acesso em: 06 jan. 2014.

FERREIRA, E. T.; COSTA, E. A. de.; SUGIZAKI, B. C.; FREIRE, V. N., BARBOSA, I. das N.; AMARAL, R. Q. G. do. Gastronomia Social: Curso de Panificação. In: XXVI Encontro de Extensão. Encontros Universitários 2017. Fortaleza, Anais... Fortaleza: UFC, 2017.

FROTA, Leandro Flavio Restrepo; COSTA, Eveline de Alencar; BASTOS, Regina Maria 
Silva; MOTA, Robson Nascimento; GARCEZ, Tereza Carlas da Nóbrega Araújo; VELHO, Liana Flor de Lima. Programa Gastronomia Social no Jardim da Gente: relato de experiência no Curso de Gastronomia de Rua. In: VII Encontro de Experiências Estudantis. Encontros Universitários 2015. Fortaleza, Anais... Fortaleza: UFC, 2015.

GOMES, Ana Carolina; MOREIRA, Marlon; PINHEIRO, Richarlisson. A gastronomia como prática pedagógica inovadora no processo ensino aprendizagem de matemática. In: IX Jornada de Iniciação Científica e Extensão. 2018. Palmas, Anais... Palmas: Instituto Federal do Tocantins, 2018.

GIANSANTE, Cláudia Cintra Bortoletto; VENELLI-COSTA; Luciano; VIEIRA, Almir Martins; DUTRA, Joel Souza. Competências coletivas e desempenho coletivo: um estudo com equipes de gastronomia. Revista Alcance - Eletrônica - vol. 22 - n. 4 - out./dez. 2015.

HOLANDA, C.; GOMES, I. Violência: Ceará teve mais de 3.5 mil homicídios dolosos em 2012. Fortaleza: Jornal o Povo, 2013. Disponível em: < http://www.opovo.com.br/app/fortaleza/2013/01/11/noticiafortaleza,2986750/violencia-cearateve-mais-3-5-mil-homicidios-dolosos-em-2012.shtml >. Acesso em: 22 fev. 2013.

KÖVESI, B.; SIFFET, C.; CREMA, C.; MARTINOLI, G. 400g: Técnicas de Cozinha. São Paulo: Companhia nacional, 2010. 568 p.

LEVIEN, Hulle Emanuela Gomes de Souza Aguilar; COSTA, Eveline de Alencar; CUNHA, Elba Cristina Barbosa da; MOTA, Robson Nascimento; BASTOS, Regina Maria Silva. Curso Gastronomia de Rua: ação promovida pelo Programa gastronomia social no jardim da gente. In: XXIV Encontro de Extensão. Encontros Universitários 2015. Fortaleza, Anais... Fortaleza: UFC, 2015.

LE BOTERF, G. Desenvolvendo a competência dos profissionais. Porto Alegre: Artmed, 2003.

LIBÂNIO, José Carlos. Didática. São Paulo: Cortez, 2017.

LORENZATO, S. Educação Infantil e percepção matemática. Campinas: Autores Associados, 2008.

SEBESS, Paulo. Técnicas de padaria profissional. São Paulo: SENAC, 2010. 319 p.

VIEIRA, Bárbara de Cássia Ribeiro; LORENZONI, Luciana de Souza; GOBBO, Sâmia D’Angelo Alcuri; BRECHIANI, Megg Cattem Moreno; Souza, Mayk Henrique. A importância da experimentação em ciências para a construção do conhecimento no ensino fundamental. Enciclopédia Biosfera, Centro Científico Conhecer - Goiânia, v.9, n.16; p 2277- 2285. 